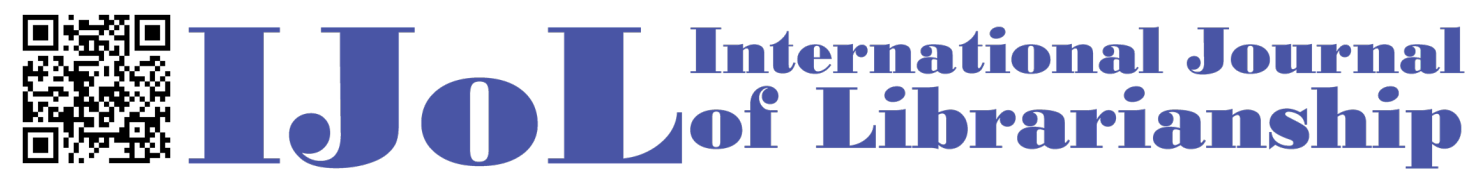

ISSN: 2474-3542 Journal homepage: https://journal.calaijol.org

\title{
FERM-ing up a Strategy for Consortial System Adoption: The Five College Consortium \& FOLIO eResource Management
}

\author{
Jack Mulvaney \\ Kathleen Berry
}

\begin{abstract}
:
The Five Colleges are committed early implementers of FOLIO and beta adopters of EBSCO's implementation and hosting services. We implemented the Agreements, eHoldings, Users, and Organizations apps in FOLIO during the summer of 2020 and will go-live on apps to replace our ILS (Integrated Library System) by summer of 2022 . Implementing the Agreements, eHoldings, Users, and Organizations apps took a significant amount of work as the management of this information and content has never been centralized or shared until now in our consortia. In this article we chronicle our path to build trust, construct a common understanding of methodology and terminology, and how we successfully migrated during a global pandemic.
\end{abstract}

To cite this article:

Mulvaney, J., \& Berry, K. (2021). FERM-ing up a Strategy for Consortial System Adoption: The Five College Consortium \& FOLIO eResource Management. International Journal of Librarianship, 6(2), 21-29. https://doi.org/10.23974/ijol.2021.vol6.2.196

To submit your article to this journal:

Go to https://ojs.calaijol.org/index.php/ijol/about/submissions 


\title{
FERM-ing up a Strategy for Consortial System Adoption: The Five College Consortium \& FOLIO eResource Management
}

\author{
Jack Mulvaney, University of Massachusetts Amherst \\ Kathleen Berry, University of Massachusetts Amherst
}

\begin{abstract}
The Five Colleges are committed early implementers of FOLIO and beta adopters of EBSCO's implementation and hosting services. We implemented the Agreements, eHoldings, Users, and Organizations apps in FOLIO during the summer of 2020 and will go-live on apps to replace our ILS (Integrated Library System) by summer of 2022. Implementing the Agreements, eHoldings, Users, and Organizations apps took a significant amount of work as the management of this information and content has never been centralized or shared until now in our consortia. In this article we chronicle our path to build trust, construct a common understanding of methodology and terminology, and how we successfully migrated during a global pandemic.
\end{abstract}

Keywords: FOLIO, eResource Management, ERM, Library Services Platform, LSP

\section{INTRODUCTION}

The Five College Consortium is composed of the Amherst campus of the University of Massachusetts and four private liberal arts colleges: Amherst College, Smith College, Hampshire College, and Mount Holyoke College. This non-profit educational consortium was established in 1965 to support the shared educational goals of the member institutions. The libraries of these institutions have shared an automated library system since the mid 1980s. The FOLIO migration is the fourth time the schools have moved to a new library services system collectively.

FOLIO is an open source library services platform. It supports applications that provide traditional library resource management functionality and soon will expand to provide new services. These applications connect to each other using a microservices architecture, allowing individual libraries to tailor their workflows for resource management and choose which apps they want to deploy. Its development is the result of collaboration between librarians, developers, and vendors from around the world. More information on the architecture of FOLIO as a system can be found on the project's main community site. (About FOLIO)

The Five Colleges are committed early implementers of FOLIO and beta adopters of EBSCO's implementation and hosting services. Our staff are active participants in the FOLIO community, providing subject matter expertise on a variety of library system functions and services. We implemented the Agreements, eHoldings, Users, and Organizations apps in FOLIO 
during the summer of 2020 . We will go-live on all remaining applications to replace our ILS by summer of 2022 .

The history of shared systems at the Five Colleges is long, save for one area of technical services work: electronic resource management (ERM). Several years ago, a subset of three libraries implemented separate, self-hosted instances of the CORAL ERM system first developed at Notre Dame. (About Us) All five libraries have used the same link resolver since 2007, but have never performed any ERM work in a single, shared system. This, combined with varying levels of staffing and focus, has led to different approaches for tracking licenses, acquisitions, discovery, and access. Even in our shared ILS, we have often taken five separate approaches to tracking the acquisition of similar or identical resources, which left us with the question of how to migrate to FOLIO structured as a single shared tenant for multiple libraries.

Reconciling these different methods of ERM, record management, and workflows provided eResource management staff at the Five Colleges with a set of unique challenges not present elsewhere in our FOLIO migration. Adding to the complexity of a system migration was the lack of a structure for unified ERM across five individual libraries. Existing consortial committees covered topics such as a shared discovery layer and resource management for specific collections bought at the consortial level. But for the majority of licensed eResources, the life cycle was handled independently at the individual libraries. Prior to the FOLIO project, no formal standing committee existed to discuss ERM as a process which meant that a structure had to be created in order to begin a successful migration (and for two schools, a new adoption) of an ERM system.

The collaboration of the humans involved in the project ultimately proved to be more important than individual understanding of how FOLIO is coded, how data flows through the system, or how it is migrated from one system to another. This focus on building bridges and trust through mutual understanding and compromise is what allowed us to successfully adopt FOLIO as an ERM system.

\section{CONSORTIAL COMMUNITY BUILDING}

To coordinate the adoption of the FOLIO ERM suite, one member from each of the Five Colleges formed the "Five Colleges FOLIO Electronic Resource Management Group" in May 2019. Existing working groups covered areas relating to circulation, inventory/metadata, and acquisitions. Affectionately known as FERM, each member represented different aspects of eResource management and the eResource life cycle as a whole, as well as a range of professional experience within the Five Colleges. An initial charge of "reaching consensus on issues related to data conversion, data management, workflows, policies and procedures...[regarding] the electronic resource life cycle within FOLIO" (Mulvaney, Berry, \& Bischof, 2019) guided our work on the assumption that any ERM adoption would take place after the main ILS migration, initially scheduled for summer 2020. This initially put us on track for the adoption of ERM in summer 2021, giving us considerable time to learn about FOLIO, plan for the migration of existing data, and to ensure a smooth adoption of a product that was still officially in beta.

This lengthy timeline allowed us to focus for the first few months on building relationships with one another, and to learn how ERM work was being done at each of the Five College libraries. It also meant we could define our working group's role within the structure of the FOLIO project 
at the Five Colleges, as well as our relationship with existing committees which overlapped in responsibility and predated the move to FOLIO. Each working group has a "lead" who meets regularly with the other leads and the Five College Libraries Systems Coordinator, who brings concerns or decisions to the FOLIO Implementation Team (FIT) which is ultimately responsible for implementing the decision on their home campus. This structure has undergone several changes since we first began to work on the implementation as we have found a need for more rapid communication and decision making. We found that it is more efficient to have leads (who are typically non-managers) make decisions and rely on institutional managers to enforce them. FERM was the first group for eResources since one that guided the adoption of the first link resolver used by the Five College libraries nearly fifteen years ago. Since then, there has not been any group which was charged with coordinating or supporting ERM among the five libraries which led to our policies and workflows drifting in five separate directions.

FERM's initial membership ranged from individuals with less than six-month experience in their role in the consortium to individuals who had been involved with the last ILS migration in 2006 and held most of the institutional knowledge that existed for their libraries' ERM actions. This varying localized and professional experiences means that we must rely on one another when troubleshooting eResources, learning about shared systems, and understanding years of context lost due to retirements or position changes at individual libraries. To strengthen a new era of direct collaboration, we specifically decided that we wanted to meet biweekly and in person on the Hampshire College campus.

Regular, in-person meetings allowed us to have a less formal setting than a web meeting or a sporadic summit, which changed the dynamic of a group of individuals who usually only had contact with one another via email or through other Five College meetings and committees. We also made use of the passing time inherent in a meeting to familiarize ourselves with one another on a personal level. Most of us would arrive at the building where our meeting was held beforehand, so we fell into a rhythm of sitting on deck chairs together in the sun admiring the campus scenery. As meetings broke up, we would routinely continue to discuss topics outside of FOLIO for 15-20 minutes as we packed our bags and walked to our cars. As lead of the working group, I initially worried that allowing our meetings to run long was bad facilitation. However, as time went on these moments of informal chit-chat about topics not on an agenda meant we were building trust in one another which directly affected our work on the FOLIO migration.

By building a cordial space where we shared local practices freely and without judgement, we could begin to tackle the difficult work of breaking down these workflows to their bare elements and rebuilding them using the newer, and very different, FOLIO ERM structure. This process of taking five unique vocabularies for managing eResources and finding a single way to approach the process was not quick, and was definitely not easy. For several months we focused on understanding how we were using our shared ILS, our link resolver and discovery layers, and in some cases a local ERM system. We tried to become familiar with how we each did the work so that we could be successful in discovering new workflows in FOLIO.

We began our work with FOLIO during its Clover release, when the system was still brand new to us and only being used by one library in the world. (EBSCO, 2019) Several working group members had only recently begun to attend FOLIO special interest group (SIG) meetings for ERM, and there was a steep uphill climb to become familiar enough with the system to use FOLIO to test how our data might go-live in a new system. Importantly, all members are practicing eResource librarians which allows us to base all decisions for how we would configure the system 
in real world experience. This meant we could take into account the shortcomings of legacy systems and current workflows to improve our overall experience in FOLIO. The lack of training or documentation for a system which remains in active, and rapid development proved a challenge, which our tight-knit group was able to move past with relative ease. By working as a team, we were able to trust each other to advocate our shared needs to the FOLIO community, to teach each other how to use less familiar parts of the system, and to support one another as we continued to do our daily work in ERM.

FERM was the first sanctioned eResource management group in the consortium and our work has proven to be a success, as of December 2021 every upgrade of the FOLIO software has been performed without major errors in the code or with our data. When asked whether we wanted to disband post implementation, the resounding answer was "no." For some, the group proved the only place where they could actively collaborate with librarians in the field of eResource management and discovery, while others found the space to finally work through legacy workflows that had been inherited but never interrogated. We requested that FERM be made a standing committee under the aegis of a future FOLIO coordinating committee so that we could continue our work in aligning policies and record management practices. This decision has meant that in the time since adoption we have continued to have productive conversations on how we can improve our system settings and how we can leverage new features being released to better carry out our work.

\section{CHANGING PERCEPTIONS OF ERM}

We began working in FOLIO ERM on the Clover release, which was the third version of FOLIO available to the public. For ERM, it consisted of the following apps: Agreements, Licenses, and eHoldings. Supporting apps included Users, Organizations, and eUsage (Boerema, 2019). Any FOLIO adopter could choose all apps or none when considering an implementation. A decision was made early on that the Five Colleges would focus on work in Agreements, eHoldings, and Organizations, with Users required for login. This specific set of apps had largely been developed prior to the Five Colleges beginning our working group and their format and philosophy were based on the needs and feedback of a group of German library consortia with supporting roles played by American libraries. This meant a serious shift for our consortium, where five discrete eResource collections are managed by institution specific staff. Each of the Five College libraries acquire, license, and manage their collections independent of each other with only a small amount of content purchased and managed at the consortial level. We were faced with the need to reconcile five different ideas of how ERM should work with the impending adoption of a single tenant of FOLIO shared across our libraries. This would mean five times as many organizations, agreements, licenses, and associated notes and details than we were used to in our more siloed ILS and individual ERM systems.

FOLIO itself also used language driven by libraries that were quite different from our own. Much of the work done to create system headings and reference data had been done before wide scale participation of the American libraries. Phrases like "alliance licenses" were used to represent the large multi-institutional licensing deals common in Europe which are not often replicated by American consortia. FERM had to then explore what we could do with the system as it stood and what configurations to settings or reference data we could make in order to ensure that our needs were best met. The FOLIO community provided a path for us to voice concerns or specific needs 
in special interest group meetings, but even among the Five Colleges there were times when one library wanted functionality to move in a different direction from another. This required a fundamental shift in our approach to the systems we use to manage eResources, as we now had a voice in the ongoing development of a product so it might meet our needs, rather than reacting to shortcomings in existing systems. Quickly, we discovered that if we worked together to come to a common understanding of what we wanted the system to do then we could more effectively advocate for our needs in SIGs which would result in faster changes to FOLIO as a product. This work strongly reflected our shared desire for making FOLIO as an open source system with flexibility to work for us, rather than having us work for the system. An early success was a collaboration between FERM members and colleagues in the consortial Acquisition Working Group to advocate for user interface improvements and a change to record structure in the Organizations app.

As a shared space between typical ILS acquisitions and an ERM system, the Organizations (or vendors) app represented a challenge when we first reviewed the app following a change to our migration timeline (outlined below.) The data structure of FOLIO was not always intuitive. Important contact data was hidden behind several layers of clicking and not optimized for when a shared email or address existed for an organization rather than a specific person. It was determined that FERM did not hold the necessary experience to make recommendations to the FOLIO community alone, so collaborative sessions were held with the Acquisitions Working Group to collect use cases for why staff need access to contact information and what they liked about how their current systems handled that data. From that data, which was agnostic of any specific legacy system and framed in terms of what was most needed rather than what fixes should be applied to FOLIO, a small group of individuals made a presentation on how current functionality in other areas of FOLIO might be leveraged to improve the user experience in the Organizations app. We were given the opportunity to present our case to the Resource Management SIG which resulted in many other libraries agreeing with the proposals we were making as they would address similar concerns they had found when testing FOLIO. A coordinated approach with user-story backed suggestions allowed the team developing that app to quickly implement new features, change how data displays, and launch the new code for the Daisy release (Mulvaney, Colglazier, \& Berry, 2019).

To orient ourselves in a new system, we began to review the fairly open and configurable reference data pick lists. Many fields could be locally configured to meet our local language needs in both the Agreements and Organizations apps. Few system defaults existed, and few values were required to drive functionality, so we had quite a bit of latitude to define values such as contact role, organization categories and roles, note titles, etc. The process of landing on singular terms for something as simple as "provider" versus "content provider" occupied the final months of 2019. In each pick list, at least one person had to sacrifice their own vocabulary for the sake of a shared system which meant we would all need to reconceptualize how we saw relationships between records and the actions we took to manage eResources. While at times it may have felt more akin to horse trading than librarianship, the process was structured in a way that everything was reviewed comprehensively before any final decision was made. Even simple terms like "vendor" were reviewed to ensure everyone remained on the same page. Again, we relied on our trust in each other to be able to concede when required to find a compromise where everyone had something to gain. This exercise of leaving behind highly individualized processes to come together in a shared system is one of our greatest achievements in FOLIO to date. 
Another hurdle was how to group content together to be managed in FOLIO. The system assumes that the user could be using ERM without orders, which we would be doing at go-live, so we had to reconcile methods of grouping resources based on how they are purchased, how they are licensed, how they are grouped by knowledgebase providers, or how they have been historically managed with a totally new structure unique to FOLIO. For a time, there was concern that we could not come to a single understanding of how to manage content, or that somehow by not doing this we had not achieved one of our shared principles to, "...standardize, streamline and improve best practices" (Bischof, 2018). As FERM continued to work out our best practices, we found that as five individual libraries with different staffing and approaches to ERM, FOLIO's flexibility would allow us to preserve unique workflows when needed, while also giving us the opportunity to align some practices with each other by sharing a single ERM system. In the end, we found that there was no "best" way to manage content across the consortium and that in some cases each library might have to manage the same resource differently depending on how it was locally licensed or how it was being paid for. While this can be viewed as a success of the system it may also be a drawback - as functionality has changed, some decisions have had to be revised, meaning that individual schools may need to perform manual cleanup after an upgrade before continuing with work. While this is unavoidable due to our status as beta partners, it has meant that we test each release of the system more carefully than many of our other more mature ERM, discovery, and access-based products.

\section{SUCCESS IN A PERIOD OF STRESS}

As we worked on our adoption of FOLIO, FERM was faced with batting against two rather unexpected curveballs. Our initial timeline of ERM following the ILS migration changed suddenly in October 2019. Due to features missing from the FOLIO minimum viable product that would prove crucial for a single-tenant multi-library consortial adoption, the Five Colleges decided that ERM would actually be migrated first, shortening our timeline from around a year and a half, to just around ten months. While nerve wracking, this timeline adjustment was ultimately viewed positively, as FERM felt surer of FOLIO as an ERM system than as a replacement for our current ILS. We also felt at that time that our ability to rely on one another meant we could navigate this sudden change and successfully adopt the product. We also began simultaneous link resolver migrations as the eHoldings app would reflect content directly from the EBSCO Knowledge Base inside FOLIO.

This change in the timeline quickened the pace of specific work such as data values for pick lists and conversations surrounding how best to manage content in FOLIO, but largely we expected to be able to more leisurely review existing records to see what we might want to automatically import to FOLIO and what we might want to manually recreate in the system. For CORAL users, FOLIO's flexibility meant that for the most part, many of our existing records could actually be imported which would allow us to focus on the edge cases which were difficult to manage in existing systems and to see if there were better ways that FOLIO could handle these resources. The remaining two libraries that were not using CORAL would then be able to manually handle a first-time adoption of an ERM system in the months following the go-live rather than needing to hit the ground running to replace existing functionality.

This process was again interrupted when a second curveball was thrown at us in March 2020, just five months out from the planned migration when we suddenly transitioned to remote 
work because of the COVID-19 pandemic. With a few months to go, we were juggling competing priorities of supporting remote learning, a large volume of patron troubleshooting, an avalanche of new eResource monograph purchases, and a timeline leading out to a go-live in late August 2020. While we originally wanted to reimagine how we were doing some of our work, it was decided that in order to actually migrate in summer of 2020, we would lessen the amount of manual record creation in favor of importing more existing content into FOLIO, and cleaning it up after go-live. We also individually pushed off our link resolver migrations by more than a year so we could dedicate time not spent supporting remote work to the FOLIO migration alone. This decision was made largely due to time constraints rather than as a review of our practices in legacy systems. The migration of "as is" data from one mature system to a system still in beta has not been without challenges. Often it has proved easier to create from the ground up using existing data sources as a guide rather than trying to replicate existing record structures in FOLIO. Had we been able to stay on our original timeline we likely would have found time to streamline more of our workflows and align more of our practices across the consortium.

While still meeting biweekly, we were able to build on the work we had done in the past two years to take stock of our current ERM practices and to configure FOLIO to launch into a CORAL-to-FOLIO migration for three of the Five College libraries. Working with the Five College Systems Coordinator and our FOLIO hosting specialists at EBSCO, we were able to draw as much relevant data out of CORAL as possible to import into FOLIO Agreements app to keep ERM moving forward in the first summer of the pandemic. This data represented perhaps half of the actual data stored in CORAL, which resulted in the need to manually recreate relationships between the Organizations and Agreements apps, and to manually enter data which did not have a specific location in FOLIO. While disappointing, this was a necessary step. The record structure of CORAL was much simpler than in FOLIO, meaning that only the simplest of records could be effectively migrated. Realistically, since FOLIO is built to include ILS functionality and integrate with some sort of knowledge base, the records which were harder to manage in CORAL would become easier in FOLIO. While the Five Colleges are still waiting to go-live with replacement functionality for our ILS, we have largely been able to move ERM into FOLIO so that once our ILS is migrated, we can begin the process of linking order and inventory content to create a unified approach to ERM across the library services platform.

In the course of our first year in FOLIO, we have spent much of our time cleaning up records imported from CORAL or manually creating records from ILS data. As we went, we found several rough patches where no fields or notes in FOLIO could adequately represent data points identified as crucial for ERM work. The flexibility of FOLIO once again afforded us an opportunity to localize settings to meet our specific needs rather than advocating for development in the FOLIO community. Consensus was reached on how to record resource URLs, authentication method, and simultaneous user limits. Continued conversations led to revisions of reference data and the introduction of best practices for naming records and recording certain content in order to comfortably work in a system which included four libraries worth of data which was visible but not actionable to any one library. We have often found that a decision made a year before cannot withstand new features and new understandings of how FOLIO behaves and a willingness to revise or start from scratch has been crucial to our continued work as a working group. 


\section{CONCLUSION}

The most important aspects of our migration to FOLIO were not successes in mapping records, loading data, or creating system pick lists, but the creation of a community within the Five Colleges for librarians working in ERM. By creating FERM and taking the time to establish trust with one another we forged a shared sense of purpose which informed every discussion and decision we have made over the last two years. That trust allowed us to standardize our ERM practices where possible while respecting the needs of individual libraries and has allowed us to have open communication to learn from each other and adapt our local workflows for the better. Our strong community allowed us to weather the timeline changes resulting from development cycles and the COVID-19 pandemic, and meant we could support one another on the migration while balancing the many competing priorities faced by librarians in 2020 and 2021. Our successful adoption of FOLIO ERM was due to this firm foundation created in the months before our timelines changed and has allowed our consortium to make shared troubleshooting, group decision making, and direct communication the norm for eResource management.

\section{References}

About FOLIO: Open Source for Libraries. (n.d.). Retrieved December 13, 2021, from https://www.folio.org/about/

About Us. (n.d.). Retrieved December 13, 2021, from http://coral-erm.org/about/

Bischof, S. (2018, September 1). Shared Five College principles. FOLIO Implementation FIT Monthly Newsletter, 1, 2. Retrieved December 13, 2021, from https://docs.google.com/document/d/1rriYfbahMxqX9SSZGg6j2mDOitMGMZErwXjX whOVWNQ/edit?usp=sharing

Boerema, C. (2019, July 4). Q2 2019 (Clover) release notes. Retrieved December 13, 2021, from https://wiki.folio.org/display/REL/Q2+2019+\%28Clover\%29+Release+Notes

EBSCO. (2019, October 21). Chalmers University of technology is the first institution to go live with FOLIO library service platform. Retrieved December 13, 2021, from https://www.ebsco.com/news-center/press-releases/chalmers-university-first-institutionto-go-live-with-folio

Mulvaney, J., Berry, K., \& Bischof, S. (2019, July 16). FERM working group charge [Google Doc Charge]. Retrieved December 13, 2021, from https://docs.google.com/document/d/1Ca0OW9OxSttWdErBiz3FpGfYCeBLWS9NrflM CMwE2UM/edit?usp=sharing

Mulvaney, J., Colglazier, S., \& Berry, K. (2019, November 6). FOLIO organizations: 5C RM SIG 11/8/19 [Google Sheets Presentation]. Retrieved December 13, 2021, from https://docs.google.com/presentation/d/11V_53JzESjJ4WiqlV6nxq0Q5x0_6anviLSS7uKwULs/edit?usp=sharing

\section{About the authors}

Jack Mulvaney works in discovery and eResources at UMass Amherst, a member of the Five 
College Consortium. He earned his BAs in Classics and History from UMass in 2018, and his MLS from Simmons University in 2020. He is currently the Lead of the Five Colleges FOLIO ERM working group.

Kathleen Berry is the Head of Information Resource Management at the University of Massachusetts Amherst. She is on the FOLIO Implementation Team for the Five Colleges Consortia and liaises with the Five Colleges ERM working group. 